\title{
DETERMINAÇÃO DA VELOCIDADE DE INFILTRAÇÃO PELO MÉTODO DO INFILTRÔMETRO DE ANEL EM SOLO DA REGIÃO AMAZÔNICA
}

Caroline Mascarenhas Ribeiro1; Francisco Carlos Lira Pessoa ${ }^{2}$;Carlos Eduardo Aguiar de Souza Costa ${ }^{3}$;Deivid Maia de Jesus ${ }^{4}$;Rayane de Nazare Martins Sales ${ }^{5}$.

1Engenheira Sanitarista e Ambiental (UFPA),(mascarenhas.carolinengsa@gmail.com) Belem, PA - Brasil.

${ }^{2}$ Doutor em Eng. de Recursos Naturais. Professor e Pesquisador (UFPA)

${ }^{3}$ Engenheiro Sanitarista e Ambiental, Doutorando no Programa de Pós-Graduação em Eng. Civil (UFPA)

${ }^{4}$ Engenheiro Sanitarista e Ambiental (UFPA)

${ }^{5}$ Engenheira Sanitarista e Ambiental (UFPA)

\section{Recebido em: 06/04/2019 - Aprovado em: 10/06/2019 - Publicado em: 30/06/2019} DOI: 10.18677/EnciBio_2019A51

\begin{abstract}
RESUMO
A infiltração se constitui como movimento no qual a água penetra verticalmente no solo pela ação da gravidade e da ação capilar, podendo ou não atingir uma zona saturada ou um aquífero. Com o crescimento populacional, os aquíferos têm se tornado cada vez mais vulneráveis a contaminações, sendo este um dos problemas mais preocupantes dentro da gestão de recursos hídricos. Dessa maneira, o objetivo geral do estudo foi calcular a velocidade de infiltração em dois pontos de medição no Campus Profissional da Universidade Federal do Pará (UFPA), buscando avaliar sua suscetibilidade a contaminação. Os ensaios foram feitos com base na metodologia do infiltrômetro por anéis. Estes aneis foram cravados até uma profundidade de 10 cm, sendo que a lâmina d'gua foi medida nos tempos de 2, 4, 6, 8, 10 e 15 minutos, permanecendo 0 último tempo até completar-se três horas de análise. Posteriormente, os dados foram aplicados na equação de Kostiakov-Lewis e submetidos aos cálculos. A classificação foi do tipo "alta" no Ensaio 1, com Velocidade de Infilração Básica (VIB) de $20 \mathrm{~mm} / \mathrm{h}$, e "muito alta" no Ensaio 2, com VIB de $180 \mathrm{~mm} / \mathrm{h}$. Comparando os ensaios, conclui-se que a taxa de infiltração é um indicador em potencial de vulnerabilidade do solo, uma vez que existem diversos fatores que podem exercer influência na quantidade de água que penetra no perfil do solo, como o grau de permeabilidade e a tipologia do mesmo, a exemplo pode-se concluir que o solo no Ensaio 2 é do tipo arenoso, facilitando possíveis contaminações.
\end{abstract}

PALAVRAS-CHAVE: águas subterrâneas; equação de kostiakov-lewis, Infiltrômetro. 


\title{
DETERMINATION OF INFILTRATION VELOCITY BY THE METHOD OF RING INFILTRATOR IN SOIL AMAZON
}

\begin{abstract}
Infiltration is a movement in which water penetrates vertically into the soil by the action of gravity and capillary action, and may or may not reach a saturated zone or an aquifer. With population growth, aquifers have become increasingly vulnerable to contamination, and this is one of the most worrying issues in water resource management. Thus, the overall objective of the study was to calculate the velocity of infiltration at two measurement points in the Professional Campus of the Federal University of Pará (UFPA), seeking to evaluate its susceptibility to contamination. The tests were done based on the infiltrater by ring methodology. These rings were drilled to a depth of $10 \mathrm{~cm}$, the water blade being measured at the times of 2, 4, 6, 8, 10 and 15 minutes, remaining the last time until three hours of analysis were completed. Subsequently, the data were applied to the Kostiakov-Lewis equation and submitted to the calculations. The classification was of the "high" type in Test 1, with Basic Infiltration Speed (VIB) of $20 \mathrm{~mm} / \mathrm{h}$, and "very high" in Test 2, with VIB of $180 \mathrm{~mm} /$ h. Comparing the tests, it is concluded that the rate of infiltration is a potential indicator of soil vulnerability, since there are several factors that may influence the amount of water entering the soil profile, such as the degree of permeability and For example, it can be concluded that the soil in Test 2 is of the sandy type, facilitating possible contaminations.
\end{abstract}

KEYWORDS: equation of kostiakov-lewis; groundwater; infiltrometers.

\section{INTRODUÇÃO}

Como recurso essencial a vida, a água é substancial em diversas atividades antrópicas. Seu ciclo possui elementos indispensáveis para a manutenção do equilíbrio natural e da urbanização, elementos deste ciclo como a infiltração, a evapotranspiração e o escoamento superficial são dependentes diretos do meio físico ao qual pertencem (OLIVEIRA et al., 2018).

A infiltração se constitui como movimento no qual a água penetra verticalmente no solo pela ação da gravidade e da ação capilar, podendo ou não atingir uma zona saturada ou um aquífero (SALES; TARGA, 2017). Este processo é importante na fase terrestre do ciclo hidrológico, uma vez que por intermédio dele é possível calcular o quanto de água precipitada penetra no solo e quanto escoa superficialmente (SOUSA; RODRIGUES, 2014).

Segundo Zaluski e Antonelli (2014) a infiltração é dependente de vários fatores, principalmente ligados a funcionalidade do solo, à sua superfície, e ao preparo e manejo do mesmo, modificando sua condutividade hidráulica e o encrostamento superficial. A característica da superfície do solo é um dos fatores determinantes no processo de infiltração, dessa maneira a manutenção da cobertura vegetal é essencial para a conservação do mesmo, devido a vegetação ser responsável pelo aumento de macroporosidade da camada superficial e da condutividade hidráulica (ZALUSKI; ANTONELLI, 2014).

O processo de infiltração é um movimento desacelerado, uma vez que após certo tempo sua velocidade torna-se constante. Este valor constante de velocidade é denominado de Velocidade de Infiltração Básica (VIB), neste momento o solo estará 
saturado (JOSÉ et al., 2018). A VIB é de suma importância em projetos de saneamento ambiental, em razão de sua grandeza ser uma das variáveis no dimensionamento da rede de drenagem e de reservatórios, bem como de estruturas de controle de erosão e processos de irrigação (FREITAS et al., 2018).

São diversos os experimentos realizados na obtenção da VIB, entre os mais utilizados destaca-se o da infiltração por anéis, por sua facilidade e prática execução (JABUR et al., 2015). Essa metodologia também é utilizada para comparar esta característica em dois ou mais locais com tipos de solos ou camadas diferentes (ROCHETA et al., 2017).

O solo se constitui como um filtro, possuindo uma capacidade de depuração, imobilizando diversas impurezas que nele são depositadas (RUGGENTHALER et al, 2016). Quando ocorre a diminuição da capacidade de suporte da terra, tem se imediatamente o aumento da densidade, a diminuição da macroporosidade e o armazenamento de água ao longo do seu perfil, correspondendo a um quadro de compactação (CUNHA et al., 2014). Tal relação, solo e lençol freático, apresenta uma forte relação, na qual possuem uma íntima independência.

Segundo Pereira Júnior et al., (2015) a infiltração de contaminantes no solo pode se tornar uma problemática de saúde pública, caso ultrapasse os limites de tolerância definidos na resolução no 396 de 03 de Abril de 2008, do CONAMA (Conselho Nacional do Meio Ambiente). A heterogeneidade no comportamento do solo e subsolo e sua capacidade de atenuação natural dos contaminantes, torna necessário a delimitação de áreas de proteção, alem de apenas determinar medidas universais de controle de contaminação. Desse modo, é imprescindível estudos de vulnerabilidade, potencial e de risco à contaminação, sobretudo nas áreas de recarga de aquíferos (PEREIRA JÚNIOR et al., 2015).

Nesse contexto, os aquíferos têm se tornado cada vez mais vulneráveis, resultando em um dos problemas mais preocupantes na gestão de recursos hídricos subterrâneos, em função de estes serem considerados reservatórios estratégicos para humanidade (FRANCO et al., 2015).

A análise da vulnerabilidade de sistemas se constitui como um indicador da capacidade de suporte ambiental, pois depende das características do ambiente natural e da sua dinâmica. Por meio de mapeamento, é possível analisar e classificar os meios em função dos diferentes graus de suscetibilidade ou vulnerabilidade (KEMERICH et al., 2011). Torna-se, possível saber se determinadas áreas são mais vulneráveis ou não, associando as intervenções antrópicas a capacidade de suporte ambiental e se constituindo como um instrumento no ordenamento do uso do solo (DAMAME et al., 2019).

Mesmo verificando a importância da análise do potencial de infiltração do solo, poucos trabalhos ainda são realizados na região Amazônica. Dessa maneira, com o intuito de incentivar trabalhos semelhantes, utilizando uma metodologia acessível, o objetivo geral do estudo foi calcular a velocidade de infiltração em dois pontos diferentes no Campus Profissional da Universidade Federal do Pará (UFPA), buscando avaliar sua suscetibilidade para poluição. Na UFPA existem áreas permeáveis, que além de contribuírem contra alagamentos decorrentes do período de chuva, podem através do experimento, avaliar potenciais de poluição decorrentes das atividades geradas na área. 


\section{MATERIAL E MÉTODOS}

$\mathrm{Na}$ Figura 1 são apresentados os procedimentos metodológicos e a ordem que ocorreram.

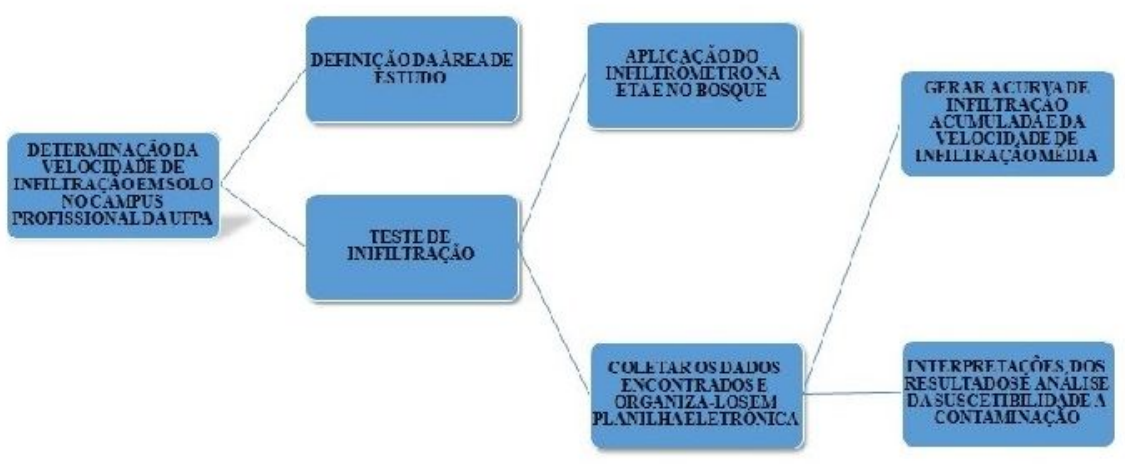

FIGURA 1 - Procedimentos metodológicos

Fonte: Os autores (2018).

\section{Área de estudo}

A pesquisa foi realizada na cidade universitária Profo ${ }^{\circ}$ José da Silveira Netto (Campus Profissional), que está localizada a margem direita do rio Guamá com coordenadas $1^{\circ} 28^{\prime} 18^{\prime \prime S}$ e 4827'09”W (Figura 2), no bairro do Guamá - Belém - Pará como mostra a Figura 2 abaixo:

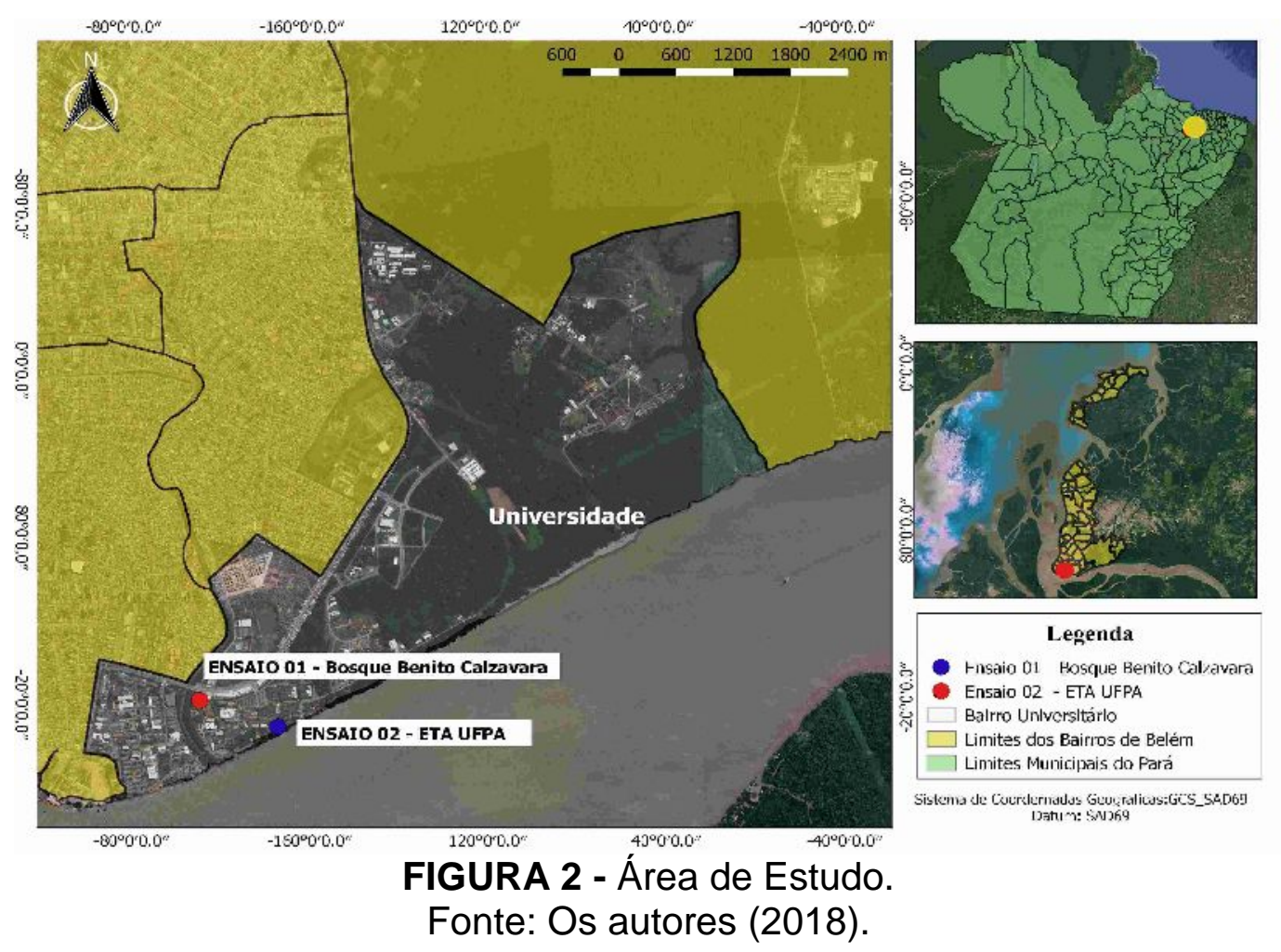


A universidade possui formação sedimentar holocênica, com planície de inundação, com superfícies argilosas orgânicas moles em espessura de 20 a 40 m, onde aproximadamente localiza-se o lençol freático, em média a 1,4 metros de profundidade. O solo é fértil devido aos sedimentos trazidos e depositados pela força da inundação.

\section{Teste do equipamento}

Inicialmente, foram realizados dois testes piloto com o equipamento para avaliar seu funcionamento no solo, um em frente ao Laboratório de Engenharia Sanitária e Ambiental (LAESA) e o outro na área onde encontra-se a Estação de Tratamento de Água (ETA).

Após os testes, foram escolhidos os dois pontos para a realização dos ensaios. O primeiro ensaio foi realizado no Bosque Benito Calzavara, em função de ser uma área próxima à Faculdade de Engenharia Sanitária e Ambiental e por isso mais fácil no transporte dos cilindros e de outras ferramentas. A área pertence ao Instituto de Tecnologia (ITEC) e é ocupada pelo Projeto Espaço ITEC, que recentemente sofreu uma urbanização, com uma consequente compactação do solo e aumento na circulação de pessoas. Este foi inaugurado em março de 2011, reunindo vários projetos tecnológicos e sustentáveis, com o objetivo de mostrar à população a importância desses espaços na Amazônia. O espaço é considerado o maior bosque do Campus apresentando passarelas para pedestres, mesas para estudos, pesquisas em várias áreas, funções de lazer e educação ambiental.

O segundo ensaio foi realizado na área da ETA da Universidade, construída em meados da década de 80 , tal escolha teve como critério a captação de água e por estar mais afastada do meio urbano. A estação é constituída pelas unidades de captação, tratamento, estação elevatória, adução, reservação e rede de distribuição, as quais abastecem os quatros principais setores da UFPA.

A Agência Nacional das Águas recomenda que os anéis possuam $25 \mathrm{~cm}$ de altura, o primeiro com $50 \mathrm{~cm}$ de diâmetro e o segundo anel com $15 \mathrm{~cm}$ de diâmetro. Porém, pela disponibilidade do equipamento, a VIB foi obtida de acordo com a metodologia de Bernardo et al. (2006) colocando dois anéis concentricamente, sendo o menor com $25 \mathrm{~cm}$ de diâmetro e o maior de $50 \mathrm{~cm}$ com altura de $30 \mathrm{~cm}$.

Os anéis devem ser cravados a uma profundidade de até $10 \mathrm{~cm}$, e a reposição da água deve ser feita sempre que o nível chegar próximo a $5 \mathrm{~cm}$ de altura da régua. $O$ anel externo funciona como um redutor do efeito de aspersão lateral da água que se espalha. Dessa maneira, a água do anel interno penetra verticalmente no perfil do solo, diminuindo a superestima da taxa de infiltração.

Primeiramente, foi feita a limpeza dos locais de ensaio utilizando uma enxada e uma pá pequena para a retirada das gramíneas e raízes. Em seguida, os anéis foram encaixados no ponto de ensaio e a terra ao redor do cilindro foi compactada para fixa-los e impedir que a água no interior do instrumento passasse. Após a afixação dos anéis, por meio de garrafas pets encheram-se as áreas dos anéis, primeiro o externo e posteriormente o interno (até o topo). Por meio de uma régua $\mathrm{e}$ um cronômetro começou-se a acompanhar a infiltração vertical. 


\section{VIB e Taxa de infiltração}

Os dados medidos foram organizados em planilha eletrônica para formular a VIB e a taxa de infiltração. Os tempos que as leituras foram realizadas foram: 2, 4, 6, 8,10 e 15 minutos, permanecendo o último tempo até completar-se três horas de análise. Assim, determinou-se a infiltração acumulada (Equação 1).

Em que:

$$
\mathrm{I}=\mathrm{k} \times \mathrm{T}^{\mathrm{a}}
$$

I = Infiltração acumulada $(\mathrm{mm})$;

$\mathrm{k}=$ Constante que representa a lâmina infiltrada no primeiro minuto, $\mathrm{em} \mathrm{cm}$;

$\mathrm{T}=$ tempo para infiltrar a lâmina de água, em min.; e, 1).

$\mathrm{a}=$ declividade da reta, determinada no local para cada tipo de solo $(0<\mathrm{a}<$

A velocidade de infiltração (VI) instantânea é a derivada da infiltração acumulada, obtida na Equação 2:

$$
\mathrm{VI}=\mathrm{dl} / \mathrm{dT} \text {, ou seja, } \mathrm{VI}=\mathrm{k} \cdot \mathrm{a} \cdot \mathrm{T}^{\mathrm{a}-1}
$$

A velocidade de infiltração média (VIM) é a divisão de I pelo tempo T:

Em que:

$$
\mathrm{VIM}=\mathrm{I} / \mathrm{T}, \mathrm{VIM}=\mathrm{k} \cdot \mathrm{T}^{\mathrm{a}} / \mathrm{T}, \mathrm{VIM}=\mathrm{k} \cdot \mathrm{T}^{(\mathrm{a}-1)}
$$

$\mathrm{VI}=$ velocidade de infiltração média, em $\mathrm{mm} / \mathrm{h}$;

$\mathrm{k}=$ Capacidade de infiltração no primeiro minuto, em $\mathrm{mm} / \mathrm{h}$;

$T$ = tempo para atingir a velocidade de infiltração instantânea, em min.; e,

$\mathrm{a}=$ declividade da reta, determinada no local para cada tipo de solo $(0<\mathrm{a}<$ 1).

Utilizando os logaritmos, temos:

$\log l=\log C+n \times \log T$

$y=\log I$ (infiltração acumulada)

$A=\log k$

$\mathrm{B}=\mathrm{a}$

$\mathrm{X}=\log \mathrm{T}$ (tempo acumulado)

Utilizou-se a regressão linear para encontrar os valores na equação linear, dada pelas Equações 4 e 5 :

$$
\mathrm{a}=B=\frac{\sum x \times \Sigma y-n \times \Sigma x y}{(\Sigma x)^{2}-\mathrm{n} \times \Sigma \mathrm{x}^{2}}
$$

Se $k=\operatorname{antilog} A$, então:

$$
A=\frac{\sum x \times \sum y-n \times \Sigma x y}{(\Sigma x)^{2}-n \times \Sigma x^{2}}
$$

$A=\log k, k=\operatorname{antilog} A$, então, $k=10^{A}$

$\mathrm{B}=\mathrm{a}$, então, $\mathrm{a}=\mathrm{B}$. 
O valor de k é encontrado aplicando o antilog A, e "a" é o próprio B. Sendo "a" o número de leituras realizada com a régua. Para obtenção da VIB, foi usada a Equação 6 de Kostiakov-Lewis, como objetivo de solucionar o problema da Tempo de infiltração básica $(\mathrm{TI})$ tender a zero:

$$
\mathrm{I}=\mathrm{k} \cdot \mathrm{T}^{\mathrm{a}}+\mathrm{VIB} \cdot \mathrm{T}
$$

Nesta situação, os parâmetros da equação de infiltração k e a são estimados pelo método de regressão. O metodo de Kostiakov-Lewis é um modelo matemático desenvolvido para modelar tempos com tendência ao infinito.

No caso da infiltração, sua velociadade tende para uma constante, ao invés de se anular. O modelo de Kostiakov-Lewis responde bem na maior parte dos solos, com grande confiabilidade. Dessa forma, foi possivel aplicar na equação os dados encontrados na ETA e no Bosque Benito Calzavara.

\section{RESULTADOS E DISCUSSÃO}

No final dos experimentos, ocorreu uma infiltração acumulada de $25 \mathrm{~mm}$ no Bosque Benito Calzavara (Tabela 1) e de $435 \mathrm{~mm}$ na ETA (Tabela 2). Observou-se no ensaio do bosque uma lâmina de $10 \mathrm{~mm}$ após 40 minutos de ensaio, ao contrário do último experimento, que leva um tempo mais rápido, de dois minutos para infiltrar uma lâmina de $40 \mathrm{~mm}$, como mostra a tabela 1 abaixo:

TABELA 1 - VIB e Infiltração no Ensaio 1.

\begin{tabular}{|c|c|c|c|c|c|c|c|c|c|}
\hline \multicolumn{10}{|c|}{ Local: Bosque Benito Calzavara (Ensaio 1) } \\
\hline \multirow{2}{*}{$\begin{array}{l}\mathrm{N}^{\circ} \text { de } \\
\text { obs. }\end{array}$} & \multicolumn{4}{|c|}{ Tempo (min.) } & \multicolumn{2}{|c|}{ Régua $(\mathrm{mm})$} & \multirow{2}{*}{$\begin{array}{l}\text { Infiltração } \\
\text { acum. } \\
\text { (mm) }\end{array}$} & \multicolumn{2}{|c|}{$\begin{array}{l}\text { Vel. de Infiltração } \\
(\mathrm{mm} / \mathrm{h})\end{array}$} \\
\hline & Hora & Dif. & Acum. & $\begin{array}{c}\text { Tempo } \\
\text { (h) }\end{array}$ & Leitura & Dif. & & $\begin{array}{c}\text { Vel. } \\
\text { instantânea }\end{array}$ & $\begin{array}{c}\text { Vel. } \\
\text { Média }\end{array}$ \\
\hline 1 & $10: 20$ & 0 & & & 140 & & & & \\
\hline 2 & $10: 22$ & 2 & 2 & 0,03 & 140 & & & & \\
\hline 3 & $10: 24$ & 2 & 4 & 0,07 & 140 & & & & \\
\hline 4 & $10: 26$ & 2 & 6 & 0,1 & 140 & & & & \\
\hline 5 & $10: 28$ & 2 & 8 & 0,13 & 140 & & & & \\
\hline 6 & $10: 30$ & 2 & 10 & 0,17 & 140 & & & & \\
\hline 7 & $10: 45$ & 15 & 25 & 0,42 & 140 & & & & \\
\hline 8 & $11: 00$ & 15 & 40 & 0,67 & 130 & 0 & 10 & 40 & 15 \\
\hline 9 & $11: 15$ & 15 & 55 & 0,92 & 130 & & 10 & 0 & 10,91 \\
\hline 10 & $11: 30$ & 15 & 70 & 1,17 & 130 & & 10 & 0 & 8,57 \\
\hline 11 & $11: 45$ & 15 & 85 & 1,42 & 127,5 & 2,5 & 12,5 & 10 & 8,82 \\
\hline 12 & $12: 00$ & 15 & 100 & 1,67 & 127,5 & & 12,5 & 0 & 7,5 \\
\hline 13 & $12: 15$ & 15 & 115 & 1,92 & 127,5 & 2,5 & 15 & 10 & 7,83 \\
\hline 14 & $12: 30$ & 15 & 130 & 2,17 & 120 & 5 & 20 & 20 & 9,23 \\
\hline 15 & $12: 45$ & 15 & 145 & 2,42 & 120 & & 20 & 0 & 8,28 \\
\hline 16 & $13: 00$ & 15 & 160 & 2,67 & 115 & 5 & 25 & 20 & 9,38 \\
\hline 17 & $13: 15$ & 15 & 175 & 2,92 & 115 & & 25 & 0 & 8,57 \\
\hline 18 & $13: 30$ & 15 & 190 & 3,17 & 115 & & 25 & 0 & 7,89 \\
\hline
\end{tabular}


No Bosque a resposta foi menos intensa (Tabela 2), contrariando as informações do estudo de Morais (2012), onde o autor comenta que a cobertura vegetal aumenta a macroporosidade dos solos, aumentando dessa maneira taxas crescentes de infiltração. No trabalho de Nunes, os resultados evidenciaram justamente isso, a taxa de infiltração era maior em solos com maior cobertura vegetal, os autores citam que este fato pode ser possível devido ao sistema radicular, o qual durante seu crescimento criam canais que favoreceram 0 deslocamento da água (NUNES et al., 2012).

TABELA 2 - VIB e Infiltração no Ensaio 2.

\begin{tabular}{|c|c|c|c|c|c|c|c|c|c|}
\hline \multicolumn{10}{|c|}{ Local: ETA (Ensaio 2) } \\
\hline \multirow{2}{*}{$\begin{array}{l}N^{\circ} \text { de } \\
\text { obs. }\end{array}$} & \multicolumn{4}{|c|}{ Tempo (min.) } & \multicolumn{2}{|c|}{ Régua (mm) } & \multirow{2}{*}{$\begin{array}{l}\text { Infiltração } \\
\text { acum. } \\
\text { (mm) }\end{array}$} & \multicolumn{2}{|c|}{$\begin{array}{c}\text { Vel. de Infiltração } \\
(\mathrm{mm} / \mathrm{h})\end{array}$} \\
\hline & Hora & Dif. & Acum. & $\begin{array}{c}\text { Tempo } \\
\text { (h) }\end{array}$ & Leitura & Dif. & & $\begin{array}{c}\text { Vel. } \\
\text { instantânea }\end{array}$ & $\begin{array}{c}\text { Vel. } \\
\text { Média }\end{array}$ \\
\hline 1 & $10: 00$ & 0 & & & 140 & & & & \\
\hline 2 & $10: 02$ & 2 & 2 & 0,03 & 100 & 40 & 40 & 1200 & 1200 \\
\hline 3 & $10: 04$ & 2 & 4 & 0,07 & 95 & 5 & 45 & 150 & 675 \\
\hline 4 & $10: 06$ & 2 & 6 & 0,1 & 80 & 15 & 60 & 450 & 600 \\
\hline 5 & $10: 08$ & 2 & 8 & 0,13 & 75 & 5 & 65 & 150 & 487,5 \\
\hline 6 & $10: 10$ & 2 & 10 & 0,17 & 65 & 10 & 75 & 300 & 450 \\
\hline 7 & $10: 15$ & 5 & 15 & 0,25 & 50 & 15 & 90 & 180 & 360 \\
\hline 8 & $10: 30$ & 15 & 30 & 0,5 & 25 & 25 & 115 & 100 & 230 \\
\hline 9 & $10: 41$ & 11 & 41 & 0,68 & 140 & & 115 & & 168,29 \\
\hline 10 & $10: 45$ & 4 & 45 & 0,75 & 120 & 20 & 135 & 300 & 180 \\
\hline 11 & $11: 00$ & 15 & 60 & 1 & 115 & 5 & 140 & 20 & 140 \\
\hline 12 & $11: 15$ & 15 & 75 & 1,25 & 140 & & 140 & & 112 \\
\hline 13 & $11: 30$ & 15 & 90 & 1,5 & 70 & 70 & 210 & 280 & 140 \\
\hline 14 & $11: 45$ & 15 & 105 & 1,75 & 40 & 30 & 240 & 120 & 137,14 \\
\hline 15 & $11: 56$ & 11 & 116 & 1,93 & 140 & & 240 & & 124,14 \\
\hline 16 & $12: 00$ & 4 & 120 & 2 & 130 & 10 & 250 & 150 & 125 \\
\hline 17 & $12: 15$ & 15 & 135 & 2,25 & 45 & 85 & 335 & 340 & 148,89 \\
\hline 18 & $12: 30$ & 15 & 150 & 2,5 & 140 & & 335 & & 134 \\
\hline 19 & $12: 45$ & 15 & 165 & 2,75 & 85 & 55 & 390 & 220 & 141,82 \\
\hline 20 & $13: 00$ & 15 & 180 & 3 & 40 & 45 & 435 & 180 & 145 \\
\hline \multicolumn{4}{|c|}{ Legenda: } & \multicolumn{6}{|c|}{ Momento em que o cilindro interno é reposto } \\
\hline
\end{tabular}

A possível resposta para essa diferença pode ser o fato de que o Bosque está em uma área mais urbanizada, instalada recentemente, e seu solo mais compactado dificulta a infiltração. Segundo o estudo de Cunha et al. (2014), quando ocorre a compactação do solo, ocorre redução da infiltração de água no perfil do solo e no comportamento da sua redistribuição e retenção, em virtude das alterações da porosidade do solo.

$\mathrm{Na}$ área da ETA grande parte do solo ainda é natural, sofreu pouquíssimas modificações, que pode representar uma porosidade maior, explicando a situação da 
infiltração na área ser mais intensa. Esses dados corroboram com o de José et al. (2018), citando a porosidade como um dos aspectos que melhor explica a capacidade de infiltração de um solo, onde velocidades de infiltração elevadas podem representar maiores espaços porosos presentes.

Outra característica que está diretamente ligada à porosidade do solo é a condutividade hidráulica, um dos parâmetros que avalia a transmissividade da terra quando o solo atinge sua saturação. Entre os experimentos, o resultado que obteve maior saturação foi o primeiro teste, da ETA, por isso seria o tipo de solo com maior condutividade e maior poder de transmissividade no mesmo. Assim como no estudo de Gonçalves e Libardi (2013), um solo como uma maior porosidade tende a apresentar um fator de condutividade hidráulica melhor do que em solos compactado.

Posteriormente, após recolher e organizar os dados em planilha eletrônica utilizou-se a função logarítmica da infiltração e do tempo acumulado, onde foram calculados o coeficiente e o expoente da equação da infiltração de Kostiakov-Lewis do tipo potencial. Dessa maneira, com a transformação logarítmica nos dados, encontraram-se os valores apresentados no quadro 1:

QUADRO 1 - Grandezas Calculadas.

\begin{tabular}{|c|c|c|}
\hline Grandezas Calculadas & Bosque Benito. C. & ETA \\
\hline Declividade da reta $(\mathrm{a}=\mathrm{B})$ & 0,7196 & 0,5088 \\
\hline Capacidade de infiltração no primeiro min. (k) & 10,6 & 180,6 \\
\hline $\mathrm{a}-1$ & $-0,28$ & $-0,49$ \\
\hline Velocidade de infiltração básica (mm/h) & 20 & 180 \\
\hline Equação da infiltração acumulada & $10,606 \cdot \mathrm{T}^{0,7196}$ & $180,6 \cdot \mathrm{T}^{0,5088}$ \\
\hline Velocidade de infiltração instantânea & $457,9 \cdot T^{(-0,28)}$ & $5513,54 \cdot T^{(-0,49)}$ \\
\hline Velocidade de infiltração média & $636,36 \cdot T^{(-0,28)}$ & $10836,04 \cdot T^{(-0,49)}$ \\
\hline Equação de Kostiakov-Lewis & $10,606 \cdot T^{0,7196}+20 . T$ & $180,6 \cdot T^{0,5088}+180 . t$ \\
\hline
\end{tabular}

Optou-se pelo modelo Kostiakov-Lewis pois este vem sendo o mais utilizado em trabalhos com este objetivo. Shaji et al. (2018) realizaram um estudo semelhante no campus do Muthoot Institute of Technology e Science (MITS) na Índia, utilizando os modelos de Horton e Kostiakov, onde os autores concluíram que o último obteve melhores resultados e ajustes quando relacionados com as características do solo.

De acordo com os gráficos das Figuras 3 e 4, curva de infiltração acumulada e curva de velocidade de infiltração, observa-se a relação inversa entre estas variáveis no decorrer do tempo, isto é, a infiltração se acumula tendendo a aumentar, enquanto a velocidade tende a diminuir e se estabilizar. 


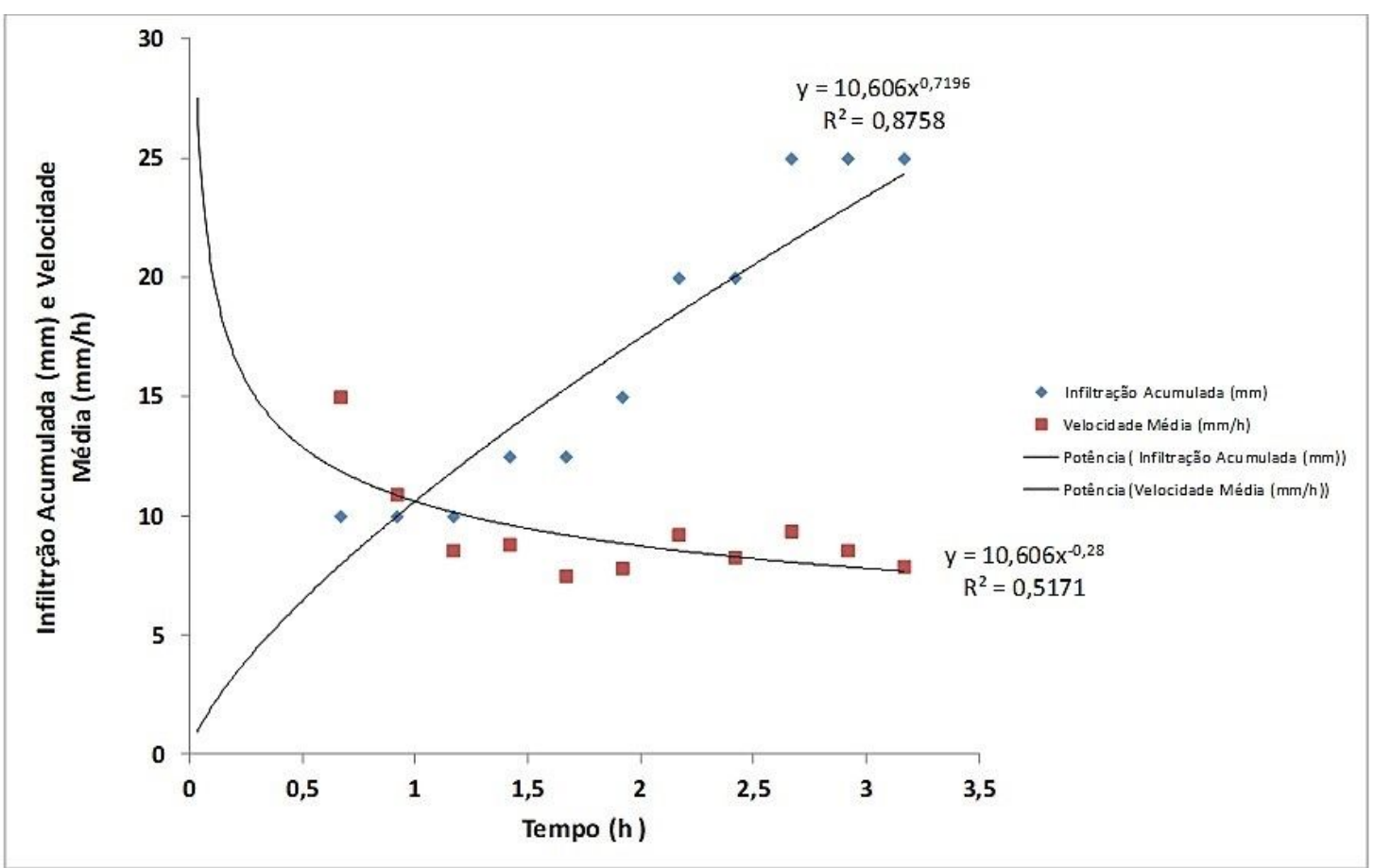

FIGURA 3 - Curvas da infiltração acumulada e VI no Bosque Benito Calzavara. Fonte: Autores, (2018).

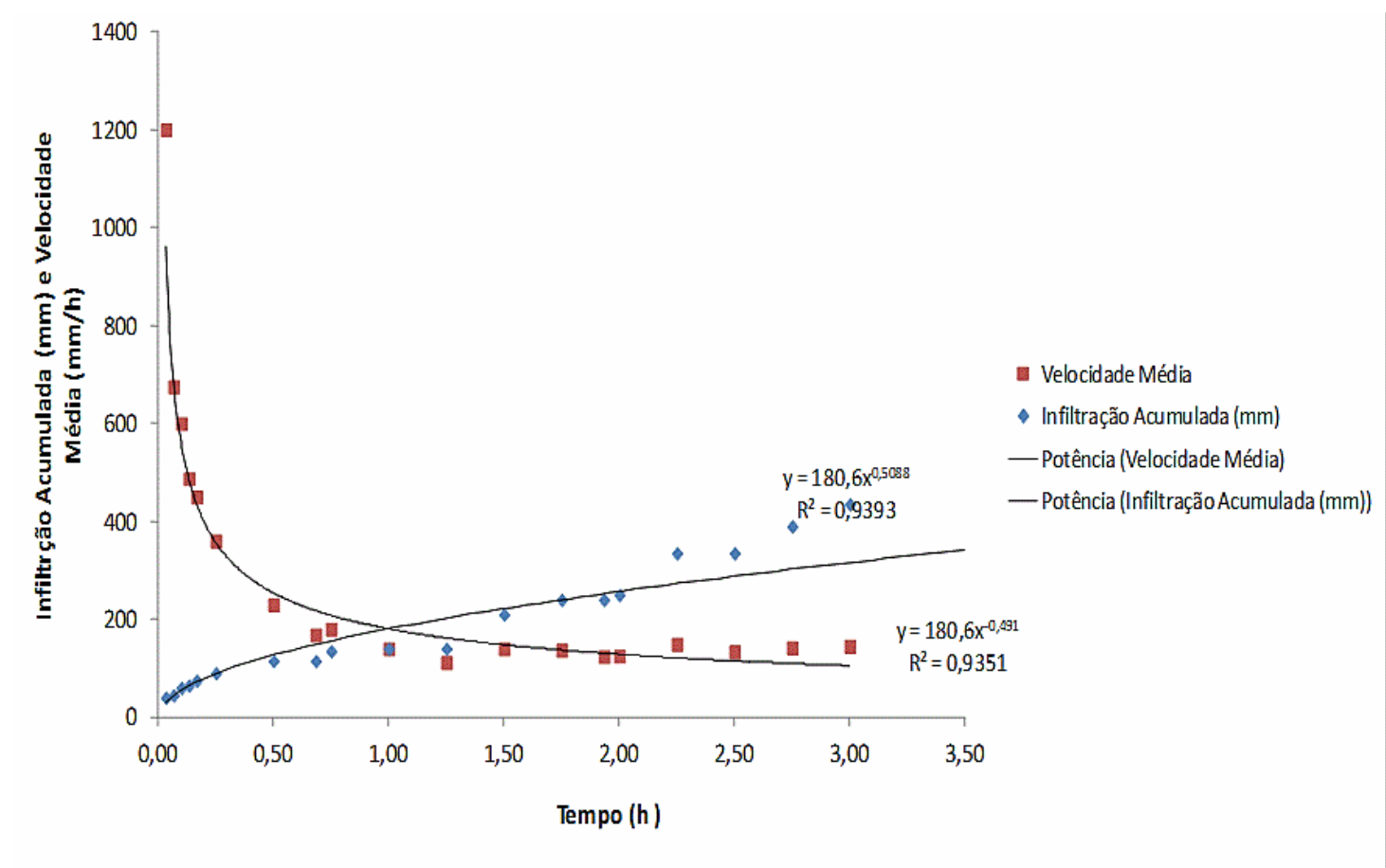

FIGURA 4 - Curvas da infiltração acumulada e VI na ETA.

Fonte: Autores, (2018).

Inicialmente, a VI no primeiro e no segundo experimento, respectivamente, foi de $40 \mathrm{~mm} / \mathrm{h}$ e $1200 \mathrm{~mm} / \mathrm{h}$, em seguida, estes valores gradativamente diminuem, 
passando o Ensaio 1 após duas horas e quarenta minutos (10h20 as 13h) para a velocidade de $20 \mathrm{~mm} / \mathrm{h}$, e o Ensaio 2 após três horas (10h a 13h) a velocidade de $180 \mathrm{~mm} / \mathrm{h}$ e mantiveram-se constantes até se caracterizar como VIB do solo.

Conforme Bernardo et al. (2006), a partir desta velocidade é possível classificar em: $>3 \mathrm{~cm} / \mathrm{h}$ (VIB muito alta), de 1,5 - 3,0 cm/h (VIB alta), 0,5 - 1,5 cm/h (VIB média) e $<0,5 \mathrm{~cm} / \mathrm{h}$ (VIB baixa). Portanto a classificação será do tipo "alta" no Ensaio 1 (Bosque), pois sua VIB de $2 \mathrm{~cm} / \mathrm{h}(20 \mathrm{~mm} / \mathrm{h})$ está entre os valores 1,5 e 3 $\mathrm{cm} / \mathrm{h}$, e tipo "muito alta" no Ensaio 2 (ETA), pois sua VIB de $18 \mathrm{~cm} / \mathrm{h}(180 \mathrm{~mm} / \mathrm{h})$ é maior que $3 \mathrm{~cm} / \mathrm{h}$. Assim, percebe-se que a VIB depende diretamente da textura e estrutura do solo.

Vale ressaltar outra característica que também influencia diretamente na VIB, a umidade no solo, que se modifica com o passar das estações do ano. No caso amazônico, mesmo sendo uma região altamente chuvosa, o experimento foi realizado já no final do período chuvoso, constatando que em outras épocas poderia apresentar VIB mais elevadas. Como exemplo desta influência observa-se os dados de Fagundes et al. (2012) que realizaram um estudo semelhante no Mato Grosso e encontraram velocidades variando entre $15 \mathrm{~cm} / \mathrm{h}$ e $4 \mathrm{~cm} / \mathrm{h}$. Já para a região nordeste (menos chuvosa do país) percebe-se mais ainda esta influência, no trabalho de Franco et al. (2015) na Bahia, os autores obtiveram taxas de infiltração muito pequenas, entre 0,18 a $1,008 \mathrm{~cm} / \mathrm{h}$.

Uma última análise que se pode realizar neste estudo é com relação a tipologia do solo. Segundo Bernardo et al. (2006) o solo pode ser classificado como: arenoso quando a velocidade variar de 25 a $250 \mathrm{~mm} / \mathrm{h}$, franco arenosa de 13 a 76 $\mathrm{mm} / \mathrm{h}$; franco-arenosa-argilosa de 5 a $20 \mathrm{~mm} / \mathrm{h}$ e franco-argilosa de 2,5 a $15 \mathrm{~mm} / \mathrm{h}$. Neste caso, no Bosque Benito Calzavara o solo pode ser dos dois últimos tipos, porém na área da ETA ele é classificado como arenoso. Conforme Cruz et al. (2010), tais solos do tipo arenosos possuem maior quantidade de macroporos do que os argilosos, correspondendo a uma maior taxa de infiltração. Tal fato pode aumentar os potenciais de poluição na área uma vez que os dois experimentos possuem o mesmo tipo de textura.

Segundo Almasri (2008), solos arenosos com altas taxas de infiltração são mais vulneráveis a contaminações, devido à baixa capacidade de retenção de água. Dessa maneira, fluidos como por exemplo chorume, águas residuais, óleos automotivos possuem um alto potencial de sofrer lixiviação e chegar no lençol freático, que além disso, é raso na maioria dos solos arenosos de zonas costeiras como no caso da ETA e pode causar contaminações na água e nas áreas adjacentes.

\section{CONCLUSÃO}

A taxa de infiltração é um potencial indicador de vulnerabilidade do solo, uma vez que existem diversos fatores que podem exercer influência na quantidade de água que penetra no perfil do solo, como o grau de permeabilidade e a tipologia do mesmo.

Nos resultados foi possível associar estas características com o grau de suscetibilidade do solo, como no ensaio na ETA, que apresentou resultados mais suscetíveis a uma potencial contaminação com uma velocidade de infiltração e infiltração acumulada igual, respectivamente a $180 \mathrm{~mm} / \mathrm{h}$ e $435 \mathrm{~mm}$. Logo, foram 
classificados como solos de VIB "muito alta" e de tipologia arenosa correspondendo a um quadro mais vulneráveis a um potencial risco de contaminação.

Comparado ao primeiro ensaio, o Bosque Benito Calzavara apresentou valores menos vulneráveis com uma VIB equivalente a $20 \mathrm{~mm} / \mathrm{h}$ e uma infiltração acumulada igual a $25 \mathrm{~mm}$ no final. Estes resultados demonstram a maior capacidade do solo de reter substâncias, se tornando, dessa maneira, menos vulnerável a alterações de alto impacto.

Sugere-se que o trabalho possa ser utilizado futuramente como base de estudo para avaliação de potenciais de vulnerabilidade de solos e águas subterrâneas, pela praticidade do método do anel e do modelo matemático de Kostiakov-Lewis que simulam o comportamento do solo como agente transportado da água assim como também de partículas benéficas ou poluentes ao meio.

\section{REFERÊNCIAS}

ALMASRI, M. N. Assessment of intrinsic vulnerability to contamination for Gaza coastal aquifer, Palestine. Journal of Environmental Management, v. 88, n. 4, p. 577-593, 2008. Disponível em: <https://www.ncbi.nlm.nih.gov/pubmed/17391837>. doi:10.1016/j.jenvman.2007.01.022.

BERNARDO, S; SOARES, A. A.; MANTOVANI, E. C. Manual de irrigação. 8. Ed. Atual. e Ampl. Viçosa: UFV, 2006. 625p.

CRUZ, J. S.; ASSIS JÚNIOR, R. N.; MATIAS, S. S. R.; CAMACHO-TAMAYO, J. H.; TAVARES, R. D. C. Spatial analysis of physical attributes and organic carbon from yellow-red alfissol with sugarcane crop. Revista Ciência e Agrotecnologia, v. 34, n. 2, p. 271-278, 2010. Disponível em: <http://dx.doi.org/10.1590/S1413$70542010000200001>$.

doi: 10.1590/S1413-70542010000200001.

CUNHA, P. D.; RITTER, L. G.; BORBA, W. F. Vulnerabilidade ambiental e áreas de infiltração máxima de água. Revista Monografias Ambientais, v. 13, n. 4, p. 37613776, $2014 . \quad$ Disponível <https://periodicos.ufsm.br/remoa/article/viewFile/14413/pdf>. doi: $10.5902 / 2236130814413$.

DAMAME, D. B.; OLIVEIRA, E. D,; LONGO, R. M. Impactos ambientais pelo uso e ocupação do solo em sub bacias hidrográficas de Campinas, São Paulo, Brasil. Acta Brasiliensis, v. $3, \quad$ n. 1, p. 1-7, 2019. Disponível em: $<$ https://www.researchgate.net/publication/330091362_Impactos_ambientais_pelo_u so_e_ocupacao_do_solo_em_sub_bacias_hidrograficas_de_Campinas_Sao_Paulo_ Brasil>.

doi: $10.22571 / 2526-4338108$.

FAGUNDES, E. A. A.; KOETZ, M.; RUDEL, N.; SANTOS, T. D.; PORTO, R. Determinação da infiltração e velocidade de infiltração de água pelo método de infiltrômetro de anel em solo de cerrado no município de Rondonópolis-MT. Revista Enciclopédia Biosfera, Centro Científico Conhecer-Goiânia, v. 8, n. 14, p. 369, 
2012.

<http://www.conhecer.org.br/enciclop/2012a/agrarias/determinacao.pdf>.

FRANCO, G.; SILVA, D.; MARQUES, E.; CHAGAS, C.; GOMES, R. Análise da Vulnerabilidade à Contaminação do Aquífero Freático e da Taxa de Infiltração do Solo da Bacia do Rio Almada e Área Costeira Adjacente-BA. Revista Brasileira de Geografia Física, v. 8, n. 3, p. 705-720, 2015. Disponível em: $<$ https://periodicos.ufpe.br/revistas/rbgfe/article/view/233387>.

FREITAS, E. F. M.; FARIAS, H. F. L.; SILVA, S. M. C.; NETO, S. A. Avaliação da Velocidade de Infiltração da Água no Solo e Uniformidade de Distribuição da Água de Irrigação por Pivô Central. Revista Brasileira de Agricultura Irrigada, v. 12, n. 1,p. 2374-2384, 2018. Disponível em: <http://www.inovagri.org.br/revista/index.php/rbai/article/view/734/pdf_449>.

doi: $10.7127 /$ rbai.v12n100734.

GONÇALVES, A. D. M. A.; LIBARDI, P. L. Análise da determinação da condutividade hidráulica do solo pelo método do perfil instantâneo. Revista Brasileira de Ciência do Solo, v. 37, n. 5,p.1074-1184, 2013. Disponível em: <http://dx.doi.org/10.1590/S0100-06832013000500007>.

doi: 10.21168/rbrh.v20n4.p937-945.

JABUR, A. S; DORNELLES, F.; SILVEIRA, A.L.L.; GOLDENFUM, J.A.; OKAWA, C.M. P.; GASPARINI, R.R.; Determinação da capacidade de infiltração de pavimentos permeáveis. Revista Brasileira de Recursos Hídricos, v. 20, n. 4, p. 937-945, $2015 . \quad$ Disponível em: <https://www.abrh.org.br/SGCv3/index.php?PUB=1\&ID=156\&SUMARIO=5119 >. doi: 10.21168/rbrh.v20n4.p937-945.

JOSÉ, J. V.; REZENDE, R.; MARQUES, P. A. A.; GONÇALVES, A. C. A.; SOUZA, R. S. Variabilidade espacial de variáveis físico-hídricas de dois Latossolos da região noroeste do estado do Paraná. Irriga, v. 17, n. 2, p. 208, 2018. Disponível em: <https://doi.org/10.15809/irriga.2012v17n2p208>.

doi: 10.15809/irriga.2012v17n2p208.

KEMERICH, P. D. C.; DESCOVI FILHO, L. L. V.; VOLPATTO, F.; SAUCEDO, É. M. Determinação da vulnerabilidade natural à contaminação da água subterrânea no bairro Nossa Senhora do Perpétuo Socorro em Santa Maria-RS. Engenharia Ambiental: Pesquisa e Tecnologia, v. 8, n. 3,p. 85-98, 2011. Disponível em: $<$ https://www.researchgate.net/publication/273934652_Determinacao_da_vulnerabili dade_natural_a_contaminacao_da_agua_subterranea_no_Bairro_Nossa_Senhora_ do_Perpetuo_Socorro_em_Santa_Maria_RS>.

MORAIS, F. Infiltration - a geomorphological variable. Caderno de Geografia, v. 22, n. 38, $\quad$ p. 73-87, $2012 . \quad$ Disponível em:<http://periodicos.pucminas.br/index.php/geografia/article/view/3753>. 
NUNES, J. A. S.; SILVEIRA, M. H. D.; SILVA, T. D.; NUNES, P. C. M.; CARVALHO, K. D. S. Velocidade de infiltração pelo método do infiltrômetro de anéis concêntricos em Latossolo Vermelho de Cerrado. Revista Enciclopédia Biosfera, v. 8, n. 15, p. 1685-1692, 2012.2 Disponível em: <http://www.conhecer.org.br/enciclop/2012b/ciencias\%20agrarias/velocidade.pdf>.

OLIVEIRA, D. B. C.; SOARES, W. A.; HOLANDA, M. A C. R. Análise de Desempenho de Modelos de Infiltração Unidimensional de Água no Solo. Revista Águas Subterrâneas, v. 32, n. 1, p. 35-42, 2018. Disponível em: $<$ https://aguassubterraneas.abas.org/asubterraneas/article/view/28947>. doi: 10.14295/ras.v32i1.28947.

PEREIRA JÚNIOR, L. C.; SOARES, H. L. T.; CASTRO, S. S. Vulnerabilidade Natural E Risco De Contaminação Do Aquífero Bauru No Município De Rio Verde - Go. Revista Águas Subterrâneas. v. 29, n. 2, p. 129-145. 2015. Disponível em: $<$ https://aguassubterraneas.abas.org/asubterraneas/article/view/27947>. doi:10.14295/ras.v29i2.27947.

ROCHETA, V. L. S.; ISIDORO, J. M. G. P.; LIMA, J. L. M. P. Infiltration of Portuguese Cobblestone Pavements-An Exploratory Assessment Using a DoubleRing Infiltrometer. Urban Water Journal, v. 14, n. 3, p. 291-297, 2017. Disponível em: < https://iahr.tandfonline.com/doi/full/10.1080/1573062X.2015.1111914>. doi: 10.1080/1573062X.2015.1111914.

RUGGENTHALER, R.; MEIBL,G.; LEITINGER, C.G.G.; ENDSTRASSER, N.; SCHOBERL, $F . ;$ Investigating the impact of initial soil moisture conditions on total infiltration by using an adapted double-ring infiltrometer. Hydrological Sciences Journal, v. $61, \quad$ n. $7, \quad$ p. 1263-1279, 2016. Disponível em: <https://doi.org/10.1080/02626667.2015.1031758>. doi: 10.1080/02626667.2015.1031758.

SALES, P. A.; TARGA, M. S. Infiltração de Água em Diferentes Usos e Ocupação dos Solos na Bacia do Rio Una em Taubaté, SP. Repositório de Ciências Ambientais, v. 1, n. 1, p. 1-13, 2017. Disponível em: <http://www.agro.unitau.br/repositorio/index.php/rca/article/view/5>.

SHAJI, A.; ASHMY M. S.; RAJU, N.; SEBASTIAN, N.; DAVID, S. J. D. Statistical Application on Infiltration Model Studies. International Journal of Current Engineering and Scientific Research, v. 5, n. 3, p. 39-42, 2018. Disponível em:<http://troindia.in/journal/ijcesr/vol5iss3part6/39-42.pdf>.

SOUSA, F. A.; RODRIGUES, S. C. Estimativa de recarga do lençol freático em solos sobre granito e gabros alcalinos na alta bacia do rio dos Boise m Iporá - GO. Ambiência, v. $10, \quad$ n. 2, p. 449-463, 2014. Disponível em: $<$ https://www.researchgate.net/publication/275044012> doi: 10.5935/ambiencia.2014.02.02

ZALUSKI, P., ANTONELI, V. Variabilidade na Infiltração da Água no Solo em área de Cultivo de Tabaco na Região Centro-Sul do Paraná. Caderno de Geografia, v.24, 
n.41, p.34-47, 2014. Disponível em: <https://doi.org/10.5752/P.23182962.2014v24n41p34>.

doi: 10.5752/P.2318-2962.2014v24n41p34 